Check for updates

Cite this: Phys. Chem. Chem. Phys., 2020, 22, 26944

Received 18th August 2020,

Accepted 8th November 2020

DOI: $10.1039 / \mathrm{d} 0 \mathrm{cp} 04392 \mathrm{c}$

rsc.li/pccp

\section{Theoretical insights into the effect of size and substitution patterns of azobenzene derivatives on the DNA G-quadruplex $\dagger$}

\author{
Kiana Gholamjani Moghaddam, (D) Goran Giudetti, (D) Wouter Sipma and \\ Shirin Faraji $\mathbb{D}$ *
}

Introducing photoswitches into the DNA G-quadruplex provides excellent opportunities to control folding and unfolding of these assemblies, demonstrating their potential in the development of novel nanodevices with medical and nanotechnology applications. Using a quantum mechanics/molecular mechanics (QM/MM) scheme, we carried out a series of simulations to identify the effect of the size and substitution patterns of three azobenzene derivatives (AZ1, AZ2 and AZ3) on the excitation energies of the two lowest excited states of the smallest photoswitchable G-quadruplex reported to date. We demonstrated that the size and the substitution pattern do not affect the ultrafast cis-trans photoiomerization mechanism of the azobenzene derivatives significantly, in agreement with the experiment. However, molecular dynamics simulations revealed that while AZ2 and AZ3 G-quadruplexes are structurally stable during the simulations, the AZ1 G-quadruplex undergoes larger structural changes and shows two ground state populations that differ in the azobenzene backbone adopting two different conformations. AZ1, with para-para substitution pattern, provides more flexibility to the whole G-quadruplex structure compared to $A Z 2$ and $A Z 3$, and can thus facilitate the photoisomerization reaction between a nonpolymorphic, stacked, tetramolecular G-quadruplex and an unstructured state after trans-cis isomerization occurring in a longer time dynamics, in agreement with the experimental findings. The QM/MM simulations of the absorption spectra indicated that the thermal fluctuation plays a more crucial role in the main absorption band of the azobenzene derivatives than the inclusion of the G-quadruplex, implying that the influence of the G-quadruplex environment is minimal. We propose that the latter is attributed to the position of the azobenzene linkers in the G-quadruplexes, i.e. the edgewise loops containing the azobenzene moieties that are located above the G-quartets, not being fully embedded inside or involved in the stacked structure. Our theoretical findings provide support to a recent study of the photoresponsive formation of photoswitchable G-quadruplex motifs. 


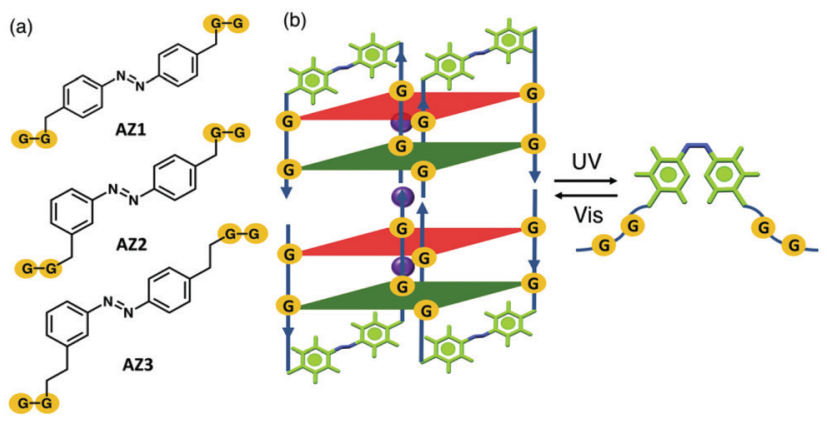

Fig. 1 (a) The structures of the three azobenzene units in the trans isomer, $G$ refers to the guanosine moieties and (b) schematic representation of the photoswitchable G-quadruplex structure with azobenzene residues (AZ1) in green color. $\mathrm{K}^{+}$cations are presented as purple spheres.

derivatives, $\mathrm{AZ1}, \mathrm{AZ2}$, and $\mathrm{AZ} 3$ as part of the backbone structure (Fig. 1a). ${ }^{23}$ This structure is a tetrameric G-quadruplex consisting of two stacked dimeric G-quadruplex units in which residues G1/G4 and G2/G5 are in syn and anti conformations along their glycosidic bonds. The size of the azobenzene derivatives, as G-quadruplex backbones, are the same for AZ1 and AZ2, but with different substitution patterns, i.e. para-para vs. para-meta, respectively. AZ3 with a para-meta substitution pattern is a double homologue of AZ2. On the basis of their findings, the antiparallel G-quadruplex can be formed for all three azobenzenes in the presence of $\mathrm{K}^{+}$ions within the G-quartets when the azobenzene linkers are in a trans conformation. In addition, spectroscopic data strongly suggest that only the G-quadruplex containing AZ1 (para-para substitution pattern) linker can enable photoswitching between a nonpolymorphic, stacked, tetramolecular G-quadruplex and an unstructured state after trans-cis isomerization of the azobenzene units (Fig. 1b). A primary mechanistic question to ask is why only AZ1 shows a defined and robust structural behavior, leading to a reversible G-quadruplex photoswitch between the folded and the unfolded G-quadruplex and not the other two, i.e. para-meta substitution pattern AZ2 and its double homologue AZ3.

Extensive theoretical studies have been performed on the photoisomerization of azobenzene and its derivatives, both in the gas phase and in solution. ${ }^{24-38}$ In addition, photoswitching of the azobenzene within DNA/RNAs has been also reported. ${ }^{39-43}$ However, to the best of our knowledge, the photoisomerization mechanism of the azobenzene derivatives and their spectroscopic properties within a G-quadruplex structure have not yet been explored computationally. For example, the effect of the size and substitution patterns of the azobenzene linkers (e.g. AZ1, AZ2, and AZ3 here) on the reversible photoswitching of G-quadruplex remained unclear. In this study, we applied mixed classical and quantum mechanical simulations to investigate the effect of different azobenzene derivatives on the spectroscopic properties of the photoswitchable G-quadruplexes. The results provide a basis for the interpretation of the experimental findings and describe the effect that the size and substitution patterns of the azobenzene units might have on the photoisomerization reaction, and whether or not there is a difference in their short-time dynamics that might potentially influence the G-quadruplex folding and unfolding that occurs in a longer time scale.
The paper is organized as follows: in Section 2, we describe the computational methods. Results from gas-phase calculations of azobenzene derivatives are presented in Section 3.1 whereas details of molecular dynamics (MD) simulations and QM/MM calculations are presented in Sections 3.2 and 3.3, respectively. Finally, Section 4 summarizes our concluding remarks.

\section{Computational methods}

The G-quadruplex structure with AZ1 (PDB code 2N9Q) ${ }^{23}$ was used as a starting structure for constructing the model system. The Parmbsc $0^{44}$ force field was selected for G-quadruplex nucleobases. Recent studies have reported that the Parmbsc0 is a valid force field for DNA simulations ${ }^{45-47}$ in particular for the simulations within the ns timescale. For atoms in the azobenzene, since it is a non-standard molecule, parameters were defined using the Generalized Amber Force Field (GAFF). ${ }^{48}$ Partial atomic charges of all azobenzene derivatives atoms were assigned with the restrained electrostatic potential $(\mathrm{RESP})^{49}$ at the HF/6-31G* level of theory. The GAFF parameters and charges for azobenzene derivatives are described in Tables S1-S4 (ESI $\dagger$ ). The G-quadruplex structure was inserted into a water box extending to $10 \AA$ buffer in each direction. To study the effect of different water models and ion parameters, three different combinations of water models and counterion parameters were used for solvation and neutralization of the negative charge of the system, respectively: group (1) Amberadapted Åqvist ${ }^{50}$ (AA) $\mathrm{K}^{+}$with the TIP3P water model ${ }^{51}$ which has been used in many simulations, group (2) Joung and Cheatham $^{52}$ (JC) $\mathrm{K}^{+}$with SPC/E water model $^{53}$ and group (3) JC KCl with the SPC/E water model which have been suggested as a safe choice for G-quadruplex MD simulations. ${ }^{54}$ The LennardJones parameters for counterions and explicit water models used in the simulations are summarized in Table S5 (ESI + ). The solvated structure was subjected to 2500 steps of energy minimization using the steepest descent algorithm. Then, the minimized structure was equilibrated under an $N V T$ ensemble (300 K) for $1 \mathrm{~ns}$ followed by $2 \mathrm{~ns} N P T$ equilibration ( $1 \mathrm{~atm}$ ) using a velocity rescaling thermostat ${ }^{55,56}$ and a Parrinello-Rahman barostat ${ }^{57,58}$ $\left(\tau_{\mathrm{T}}=0.1 \mathrm{ps}, \tau_{\mathrm{P}}=1 \mathrm{ps}\right)$. The cut off for van der Waals and electrostatic interactions was set to $10.0 \AA$. The long-range electrostatic interactions were calculated using the particle mesh Ewald (PME) method ${ }^{59}$ and the LINCS algorithm ${ }^{60}$ was used to fix all bonds. Finally, the MD production run was performed in an NPT ensemble for 200 ns. Furthermore, the PDB code 2N9Q was adapted for G-quadruplex structures containing AZ2 and AZ3, and a similar simulation setup has been used for their MD production runs. All MD simulations were performed using the GROMACS 2018.2 package. ${ }^{61}$ Additionally, the conformational space of the G-quadruplex in the trajectory was clustered using the GROMOS algorithm. ${ }^{62}$ The 90 structures from each MD production run (total of 270 configurations) were selected for the subsequent $\mathrm{QM} / \mathrm{MM}$ simulations. The snapshots were extracted by sampling the MD trajectories every $2.2 \mathrm{~ns}$ in a duration of 2-200 ns. 
The QM/MM simulations were carried out using the Q-Chem electronic structure program ${ }^{63,64}$ using structures from the MD simulations and the Parmbsc0 point charges. The interactions between QM and MM atoms were defined using the electrostatic embedding scheme ${ }^{65}$ in which the partial charges of MM atoms were used in the QM Hamiltonian as a one-particle operator. Hydrogen link-atoms were used to cap the dangling bonds when the QM and MM regions were separated. To avoid over-polarization, the charges on $\mathbf{M M}_{1}$ atoms are evenly distributed in the adjacent bound MM atoms such that the total charge is conserved. The QM region includes one azobenzene residue in the G-quadruplex structures. The rest of the G-quadruplex, three potassium ions, counterions and water molecules were considered in the MM region presented by fixed atomic point charges. The vertical excitation energies were calculated at the timedependent density functional level of theory (TD-DFT) ${ }^{66}$ using the $\omega \mathrm{B} 97 \mathrm{X}-\mathrm{D}$ functional and the cc-pVDZ ${ }^{67}$ basis set.

In order to identify the effect of the size and substitution pattern on the photoisomerization mechanisms of the azobenzene derivatives, quantum chemical calculations were performed for both isolated cis and trans isomers of azobenzene derivatives, along the trans-cis isomerization reaction coordinate using the spin-flip TDDFT (SF-TDDFT) method. Despite numerous studies on the photoisomerization reaction of isolated azobenzenes, ${ }^{24-38}$ to the best of our knowledge, the photoisomerization mechanism of azobenzene with different substitution patterns, as those considered here, has not yet been explored, in particular using the SF-TDDFT method. The ground-state geometries of AZ1, AZ2 and AZ3 were optimized in the gas phase with DFT $^{68,69}$ using the $\omega$ B97X-D exchangecorrelation (xc) functional ${ }^{70}$ along with the cc-pVDZ basis set including Grimme's dispersion correction. ${ }^{71}$ The vertical excitation energies of the $S_{1}$ and $S_{2}$ excited states and the corresponding excited-state optimized geometries were calculated using spin-flip TDDFT (SF-TDDFT)/cc-pVDZ employing the $\omega$ B97X-D and B5050LYP functionals. Relaxed potential energy surface (PES) scans were performed for the ground state $\left(\mathrm{S}_{0}\right)$ at the SF-TDDFT(B5050LYP)/cc-pVDZ level of theory that is constrained geometry optimizations by fixing the CNNC dihedral angle in the azobenzene derivatives over a range of $0-180^{\circ}$. In addition, minimum-energy crossing points (MECPs) between $\mathrm{S}_{1} / \mathrm{S}_{0}$ and $\mathrm{S}_{2} / \mathrm{S}_{1}$ were located using the branching plane updating method $^{72}$ at the SF-TDDFT(B5050LYP)/cc-pVDZ level of theory. An effective state-tracking algorithm, based on a maximum-overlap criterion as implemented in the Q-Chem program suite, ${ }^{63,73}$ is used to check the spin-contamination problem in SF-TDDFT calculations. All quantum mechanical calculations have been performed using the Q-Chem electronic structure program. ${ }^{63,64}$ Cartesian coordinates of all the relevant structures are given in the ESI. $\dagger$

\section{Results and discussion}

3.1 Photoisomerization reactions of AZ1, AZ2 and AZ3 derivatives in the gas phase

In order to understand how the size and substitution patterns of the azobenzene unit can affect its photoisomerization reactions, we begin by analyzing the ground $\left(\mathrm{S}_{0}\right)$ and excited $\left(S_{1}\right.$ and $\left.S_{2}\right)$ states potential energy surfaces (PESs) of the isolated azobenzene derivatives. We applied the SF-TDDFT method to explore potential energy surfaces of the AZ1, AZ2 and AZ3 along photoisomerization reactions (CNNC dihedral), by locating critical geometries of the $S_{0}, S_{1}$ and $S_{2}$, such as various minima, transition states, and MECPs between $S_{1} / S_{0}$ and $S_{2} / S_{1}$. The geometry parameters including CNNC dihedral $(\phi)$, two NNC bond angles $(\theta)$ and $\mathrm{NN}$ bond length are summarized in Table 1.

The ground state optimized geometries of para-substituted and meta-substituted azobenzene derivatives (AZ1, AZ2 and AZ3) are in good agreement with experimental results ${ }^{74-77}$ as well as previous theoretical studies ${ }^{78-80}$ reported for azobenzene without substitution. For the $\mathrm{S}_{1}$ minimum structure, previous theoretical studies using the complete active space self-consistent field (CASSCF) methods ${ }^{37,79-82}$ showed that the optimized CNNC dihedral angle $(\phi)$ is $180^{\circ}$ for the trans isomer of the azobenzene molecule, respectively. In contrast, the previous SF-TDDFT work ${ }^{78}$ using the BHHLYP functional showed a non-planar geometry for the trans $\mathrm{S}_{1 \mathrm{~min}}\left(\phi=143.8^{\circ}\right)$. Our results indeed revealed a nonplanar $\mathrm{S}_{1 \text { min }}$ for all three azobenzene derivatives studies here, with the corresponding CNNC dihedral, for trans isomer, of being $133.4^{\circ}, 135.2^{\circ}$ and $136.1^{\circ}$ for AZ1, AZ2 and AZ3, respectively. Since the $S_{1}$ excited state corresponds to an $n-\pi^{*}$ transition and the triplet reference state in SF-TDDFT includes the HOMO $(\pi)$ and LUMO $\left(\pi^{*}\right)$, the SF results cannot describe the $S_{1}$ state properly and show different results compared to the CASSCF methods. As is clear from Table 1 , the geometry optimizations of the $\mathrm{S}_{1}$ state starting from trans and cis isomers, are both converged to the same local minimum for AZ1 (2.44 eV energy), and to slightly different local minima for AZ2 $(2.43 \mathrm{eV}$ for trans and $2.42 \mathrm{eV}$ for cis). In contrast, the $\mathrm{S}_{2}$ geometry optimizations of the trans and cis isomers converged to different local minima; i.e. the trans isomers converged to a planar structure $\left(\phi=180^{\circ}\right)$, while the cis isomers converged to a non-planar structure with $\phi$ of $92.9^{\circ}$. The $\mathrm{S}_{2}$ optimized geometries obtained here are in good agreement with CASSCF results. ${ }^{30,83}$

The vertical excitation energies of the lowest two singlet states $\left(\mathrm{S}_{1}\right.$ and $\left.\mathrm{S}_{2}\right)$ for azobenzene derivatives obtained by SF-TDDFT (various functionals), as well as experimental and previous theoretical results using wave function based methods are summarized in Table 2. The CASPT2//CASSCF results ${ }^{30}$ show the closest agreements with the experimental results. ${ }^{84,85}$ Comparing the performance of SF-TDDFT results with wave function-based methods, it is evident that functionals containing a larger amount of HartreeFock exchange, i.e. the B5050LYP functional with 50\% HartreeFock exchange, provide a closer agreement with the computed CASPT2//CASSCF(12-14)/6-31G* values (difference of $\approx 0.5$ for both $S_{1}$ and $S_{2}$ ). It should be pointed out that in general postHartree Fock methods are especially sensitive to the nature of the basis set and including the polarization and diffusion functions can influence the vertical excitation energies of $S_{1}$ and $S_{2}$ states. Note that for all levels of theories, the order of the states remains the same; $S_{1}$ being the dark $\left(n \pi^{*}\right)$ state and $S_{2}$ being the bright $\left(\pi \pi^{*}\right)$ state for all cis and trans isomers studied here. 
Table 1 Optimized geometry parameters of the $\mathrm{S}_{0}, \mathrm{~S}_{1 \text { min }}, \mathrm{S}_{2 \text { min }}$, and $\mathrm{MECPs} \mathrm{Cl}_{\mathrm{S}_{0} / \mathrm{S}_{1}}$ and $\mathrm{Cl}_{\mathrm{S}_{1} / \mathrm{S}_{2}} . \Delta E$ (in eV) refers to the energies relative to the $\left(\mathrm{S}_{0 \text { min }}\right.$ ) of the trans isomer. Bond length $d_{N=N}$ is given in angstroms $(\AA)$, and bond angles $\theta$ and dihedral angle $\phi$ are given in degrees. Note that the experimentally reported parameters ${ }^{74-76}$ are related to the azobenzene without substitution

\begin{tabular}{|c|c|c|c|c|c|c|c|c|c|}
\hline Molecule & Geometry & \multicolumn{4}{|l|}{ trans } & \multicolumn{4}{|l|}{ cis } \\
\hline \multirow[t]{4}{*}{$\mathrm{AZ1}$} & $\mathrm{S}_{0}$ & 179.5 & $114.4 / 114.6$ & 1.247 & 0 & 6.8 & $122.6 / 122.6$ & 1.241 & 0.74 \\
\hline & $\mathrm{S}_{2 \min }$ & 179.7 & $112.8 / 112.9$ & 1.305 & 3.73 & 92.9 & $125.8 / 125.8$ & 1.248 & 2.94 \\
\hline & $\mathrm{CI}_{\mathrm{S}_{1} / \mathrm{S}_{0}}$ & 92.5 & $119.4 / 138.4$ & 1.243 & 2.32 & 92.2 & 119.3/138.4 & 1.243 & 2.32 \\
\hline & $\mathrm{CI}_{\mathrm{S}_{2} / \mathrm{s}_{1}}$ & 179.8 & $114.7 / 114.6$ & 1.329 & 3.14 & 45.9 & $117.4 / 117.3$ & 1.468 & 4.31 \\
\hline & $\mathrm{S}_{2 \min }$ & 180.0 & $112.7 / 114.3$ & 1.323 & 3.61 & 92.9 & $125.3 / 126.6$ & 1.247 & 2.92 \\
\hline & $\mathrm{CI}_{\mathrm{S}_{1} / \mathrm{S}_{0}}$ & 92.5 & $119.3 / 139.2$ & 1.242 & 2.32 & 92.0 & 119.2/137.2 & 1.245 & 2.30 \\
\hline & $\mathrm{CI}_{\mathrm{S}_{2} / \mathrm{S}_{1}}$ & 180.0 & $114.2 / 114.3$ & 1.331 & 3.15 & 53.2 & $118.2 / 115.6$ & 1.487 & 4.32 \\
\hline \multirow[t]{2}{*}{ AZ3 } & $\mathrm{S}_{0}$ & 179.2 & $114.5 / 114.4$ & 1.247 & 0 & 6.3 & $122.4 / 122.5$ & 1.241 & 0.72 \\
\hline & $\mathrm{S}_{1 \min }$ & 136.1 & $127.2 / 127.1$ & 1.241 & 2.41 & 135.7 & $127.0 / 127.1$ & 1.242 & 2.42 \\
\hline & & 180 & $113.9 / 113.9$ & 1.247 & - & & & & \\
\hline
\end{tabular}

Table 2 Vertical excitation energies of $\mathrm{S}_{1}$ and $\mathrm{S}_{2}$ states for AZ1, AZ2 and AZ3. The energies are given in eV. Note that ref. 28, 33, 72, 75 and 77 refer to the azobenzene without substitution

\begin{tabular}{|c|c|c|c|c|c|}
\hline \multirow[b]{2}{*}{ Molecule } & \multirow[b]{2}{*}{ Method } & \multicolumn{2}{|c|}{ trans } & \multicolumn{2}{|l|}{ cis } \\
\hline & & $\mathrm{S}_{1}$ & $\mathrm{~S}_{2}$ & $\mathrm{~S}_{1}$ & $\mathrm{~S}_{2}$ \\
\hline \multirow[t]{2}{*}{ AZ1 } & SF- $\omega B$ 97X-D/cc-pVDZ & 3.09 & 3.49 & 3.02 & 4.34 \\
\hline & SF-B5050LYP/cc-pVDZ & 3.07 & 3.73 & 3.05 & 4.40 \\
\hline \multirow[t]{2}{*}{ AZ2 } & SF- $\omega B 97 X-D / c c-p V D Z$ & 3.08 & 3.54 & 3.04 & 4.36 \\
\hline & SF-B5050LYP/cc-pVDZ & 3.05 & 3.78 & 3.06 & 4.40 \\
\hline \multirow[t]{2}{*}{ AZ3 } & SF- $\omega B$ 97X-D/cc-pVDZ & 3.07 & 3.53 & 3.02 & 4.34 \\
\hline & SF-B5050LYP/cc-pVDZ & 3.05 & 3.77 & 3.07 & 4.42 \\
\hline \multirow[t]{4}{*}{ Azobenzene } & 5SA-CASSCF $(6,6) / 6-31 \mathrm{G}^{86}$ & 3.08 & 5.80 & 3.77 & 5.99 \\
\hline & CASPT2//CASSCF $(12-14) / 6-31 G^{* 30}$ & 2.53 & 4.23 & 2.72 & 4.49 \\
\hline & SA3-CAS $(10,8) / 6-31 G^{*} / 6-31 G^{35}$ & 3.24 & - & 3.36 & - \\
\hline & MR-CISD $^{81}$ & 3.11 & 5.39 & 3.95 & 6.12 \\
\hline Azobenzene & Experiment (gas phase) ${ }^{84,85}$ & 2.82 & 4.12 & 2.92 & 4.68 \\
\hline
\end{tabular}

The photoisomerization dynamics are typically controlled by the energies of the minima of the various surfaces, various conical intersection seams and MECPs along these seams. Here we determined two MECPs that play a crucial role in the transcis photoizomerization of the azobenzene derivatives studies here, namely, CI between $\mathrm{S}_{1}$ and $\mathrm{S}_{2}\left(\mathrm{CI}_{\mathrm{S}_{2} / \mathrm{S}_{1}}\right)$ and CI between $\mathrm{S}_{1}$ and $\mathrm{S}_{0}\left(\mathrm{CI}_{\mathrm{S}_{1} / \mathrm{S}_{0}}\right)$.

$\mathbf{C I}_{\mathbf{S}_{2} / \mathbf{s}_{1}}$. The trans and cis $\mathrm{S}_{0 \text { min }}$ geometries served as the starting point for the corresponding MECP optimization calculations. The optimized geometric parameters and their relative energies with respect to $S_{0 \text { min }}$ of the trans isomer are listed in Table 1. Starting from the trans isomers, the optimization converged to a minimum, $\mathrm{CI}_{\mathrm{S}_{2} / \mathrm{S}_{1}}$, with an energy of $3.15 \mathrm{eV}$, and a planar structure $\left(\phi=180^{\circ}\right)$ with structural parameters being very similar to those obtained for the $\mathrm{S}_{2 \text { min }}$ : the CNNC dihedral difference of $\approx 0.0^{\circ}$, NNC angle difference of $1.9^{\circ}$, but $0.59 \mathrm{eV}$ higher in energy. The latter indicates that $\mathrm{CI}_{\mathrm{S}_{2} / \mathrm{S}_{1}}$, for all three azobenzenes considered here, is located in the close vicinity of the $\mathrm{S}_{2 \mathrm{~min}}$. However, starting from the cis isomers, we converge to $\mathrm{CI}_{\mathrm{S}_{2} / \mathrm{S}_{1}}^{\prime}$ minima, with energies being around $0.8 \mathrm{eV}$ lower than that of the Franck-Condon (FC) point of the corresponding cis isomers and around $1.4 \mathrm{eV}$ higher than that of the corresponding $\mathrm{S}_{2 \mathrm{~min}}^{\prime}$. Additionally, the structural parameters substantially differ from the corresponding $\mathrm{S}_{2 \text { min }}^{\prime}$; e.g., the CNNC dihedral angles for $\mathrm{CI}_{\mathrm{S}_{2} / \mathrm{S}_{1}}^{\prime}$ are $45.9^{\circ}, 53.2^{\circ}$ and $52.1^{\circ}$ for AZ1, AZ2 and AZ3, respectively, while the dihedral angle of $\mathrm{S}_{2 \text { min }}^{\prime}$ is around $93^{\circ}$ for all three derivatives.

$\mathbf{C I}_{\mathbf{S}_{1} / \mathbf{s}_{\mathbf{0}}}$. The trans and cis $\mathrm{S}_{0 \text { min }}$ geometries served as the starting point for the corresponding MECP optimization calculations. Interestingly, both trans and cis optimizations converged to the same $S_{1} / S_{0}$ crossing points with a dihedral angle of around $92^{\circ}$ and energy of around $2.3 \mathrm{eV}$ (see Table 1). Please note that $\mathrm{CI}_{\mathrm{S}_{1} / \mathrm{S}_{0}}$ is around $0.13 \mathrm{eV}$ lower than the $\mathrm{S}_{1 \mathrm{~min}}$, but with different dihedral angles $\left(92^{\circ}\right.$ vs. $\left.135^{\circ}\right)$.

To shed further light on the cis-trans photoizomerization, the PESs for the $S_{0}, S_{1}$ and $S_{2}$ along the CNNC dihedral angle $(\phi)$ for AZ1 are depicted in Fig. 2. Analogous figures for AZ2 and AZ3 can be found in Fig. S2 (ESI $\dagger$ ). It is evident that the $\mathrm{S}_{0}$ PES has two minima connected through the transition state with $1.8 \mathrm{eV}\left(41.51 \mathrm{kcal} \mathrm{mol}^{-1}\right)$ energy barrier at $\phi=90^{\circ}$. This large barrier excludes the thermal cis-trans isomerization as a plausible reaction and further confirms that the photoisomerization being the operative mechanism. After photo-excitation to the bright $\mathrm{S}_{2}$ state, the trans isomer quickly relaxes to the first excited state $\left(\mathrm{S}_{1}\right)$ passing the $\mathrm{CI}_{\mathrm{S} 2 / \mathrm{S} 1}$ (at $\phi=180^{\circ}$ ), through which internal conversion occurs. 


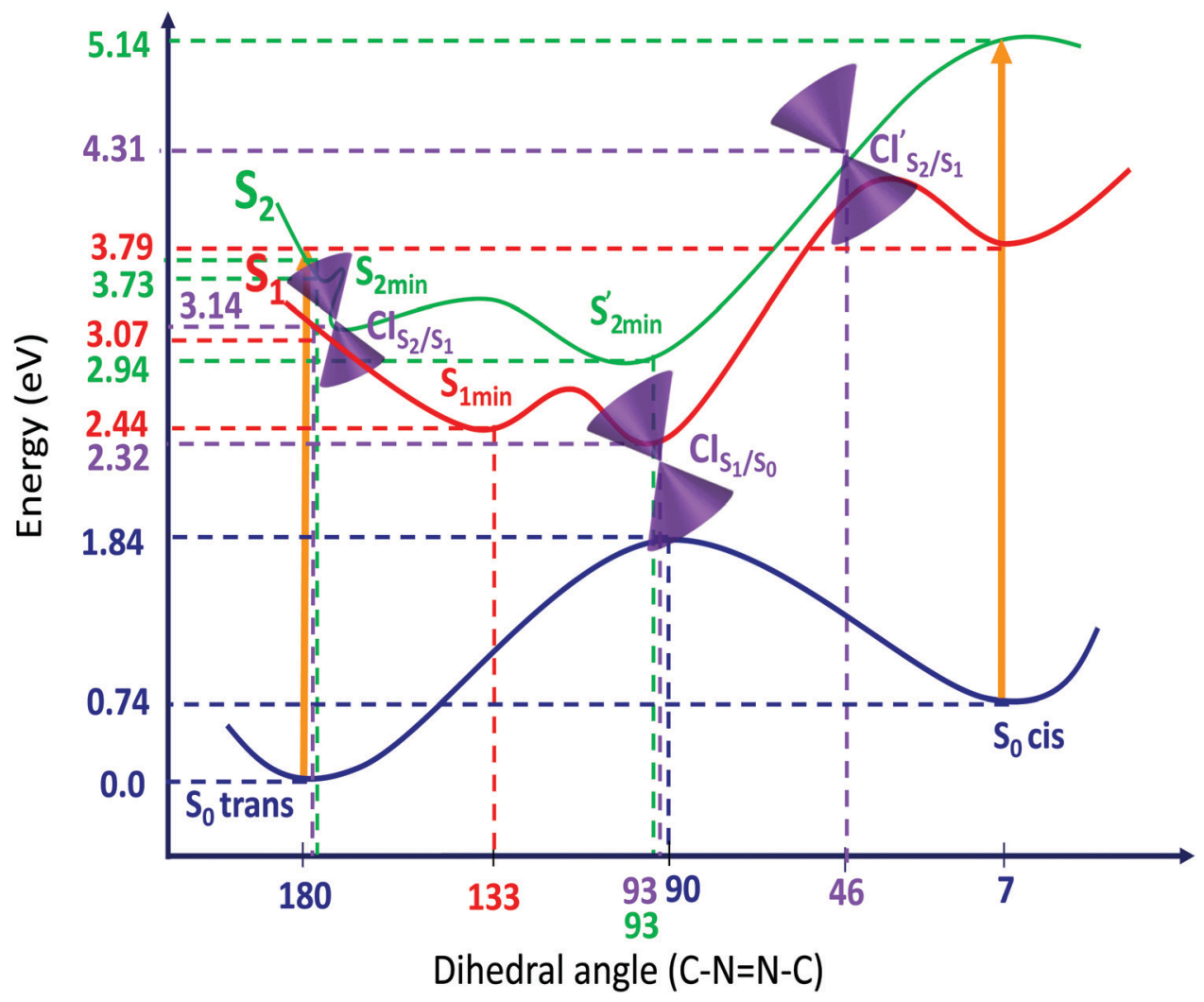

Fig. 2 Schematic representation of the PESs of AZ1 photoisomerization mechanism as function of the CNNC dihedral angle. The ground state ( $\mathrm{S}_{0}$ ), first $\left(S_{1}\right)$ and second $\left(S_{2}\right)$ excited states are shown in blue, red and green. The $S_{0}$ curve is a PES scan along the dihedral angle obtained from SF-B5050LYP/ccpVDZ. The $S_{1}$ and $S_{2}$ curves are obtained through a connection of the excited states optimized geometries and MECPs (shown in purple) calculated at the SF-B5050LYP/cc-pVDZ level of theory.

This behaviour is typical when the CI is accessible from the FC region without significant energy barriers which is the case here. From this critical point, the system undergoes vibrational relaxation towards $\mathrm{S}_{1 \text { min }}$ and from there evolves directly towards $\mathrm{CI}_{\mathrm{S} 1 / \mathrm{S} 0}$ (at $\phi=92^{\circ}$ with an energy of $2.32 \mathrm{eV}$ for AZ1/ $\mathrm{AZ} 2$ and $2.28 \mathrm{eV}$ for AZ3), without significant energy barriers (keeping in mind the excess vibrational energy after photoexcitation), which triggers an ultra-fast internal conversion process and provides a funnel of fast access to the ground state, in which the system can evolve either to the $\mathrm{S}_{0}$ of the cis or the trans isomer. A similar photoconversion mechanism occurs upon photoexcitation of the cis isomer of azobenzene derivatives, namely, after internal conversion through $\mathrm{CI}_{\mathrm{S}_{2} / \mathrm{S}_{1}}^{\prime}$, at $45.9^{\circ}, 53.2^{\circ}$ and $52.1^{\circ}$ for AZ1, AZ2 and AZ3, respectively, the system will undergo a vibrational relaxation directly towards $\mathrm{CI}_{\mathrm{S} 1 / \mathrm{s} 0}$ (at about $92^{\circ}$ ), that act again as a doorway for an ultrafast internal conversion to the ground state, on which the system can again evolve to the $\mathrm{S}_{0}$ of the cis or the trans isomer. The similar photodynamics observed for the AZ1, AZ2, and AZ3 derivatives indicate that the size and the substitution pattern does not affect the ultra-fast cis-trans photoiomerization mechanism of the azobenzene unit significantly. The latter is in line with the experimental observation, in which all three AZ1, AZ2, and AZ3 undergo photoisomerization and G-quadruplex formation.

\subsection{MD simulations}

Previous MD simulations on G-quadruplex structures indicated the role of different ion parameters and water models on the simulation results. ${ }^{54,87}$ In order to determine the optimal parameters for our system, we used three parameter combinations, i.e. $\mathrm{AAK}^{+}, \mathrm{JCK}^{+}$and $\mathrm{JCKCl}$ as described in Section 2. Throughout the following discussion, we compare the effect of these parameters on the movement of $\mathrm{K}^{+}$ions within the channel and structural stability of the G-quadruplexes during 200 ns MD production runs.

AZ1 simulations. Fig. 3a shows representative structures obtained from the clustering analysis of the three simulations that differ from one another in their ion/water parameters (see Section 2). It is clear that, for the AZ1-AAK ${ }^{+}$simulation, one of the $\mathrm{K}^{+}$cations escapes from the G-quadruplex channel into bulk water. Inspection of MD trajectories shows that at the beginning of the simulation, the top $\mathrm{K}^{+}$moves up from the channel and stays above the upper G-quartet. Afterwards, at around 8 ns simulation, the middle $\mathrm{K}^{+}$ion occupies the empty coordination site of the top $\mathrm{K}^{+}$resulting in the escape of the $\mathrm{K}^{+}$ by passing through the upper G-quartet. The position of the middle ion remains unoccupied during the rest of the simulation time (see Fig. S3 for further details, ESI $\dagger$ ). In the case of AZ1-JCK ${ }^{+}$, an ion movement is observed at $26 \mathrm{~ns}$ in which the bottom ion leaves its position and then the middle ion replaces 
(a)

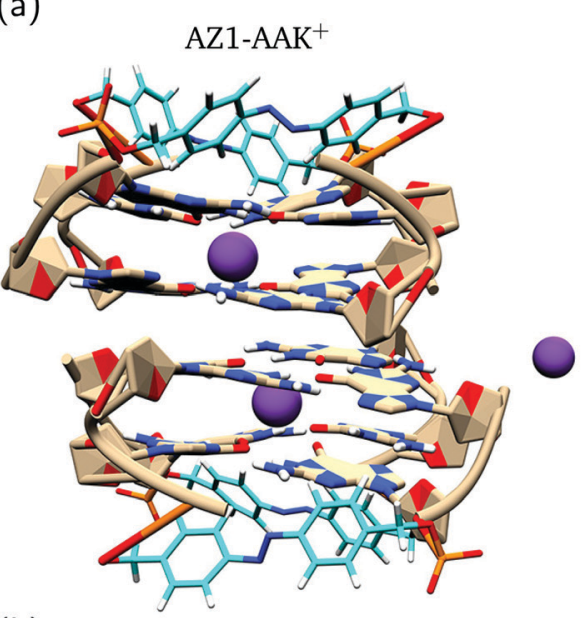

(b)

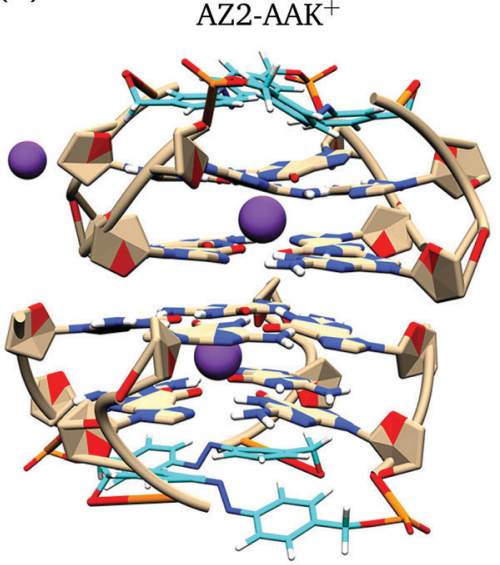

(c)

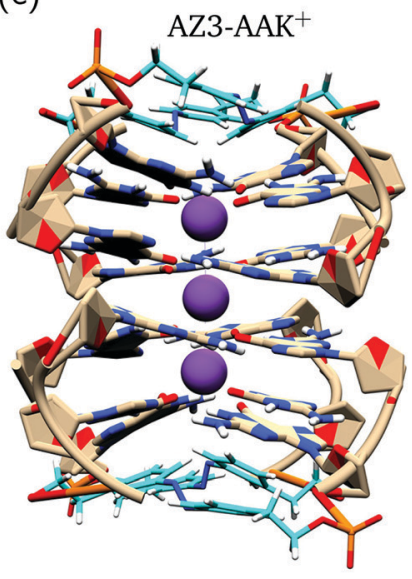

AZ1-JCK

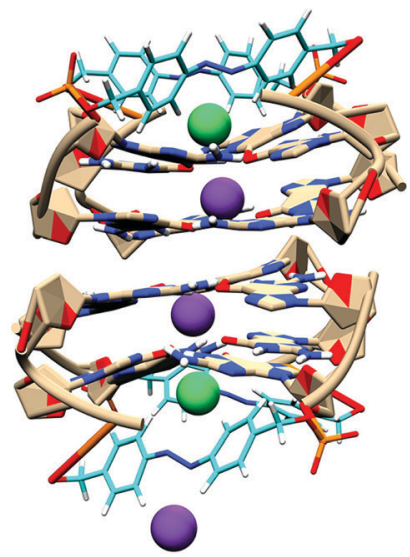

AZ2-JCK ${ }^{+}$
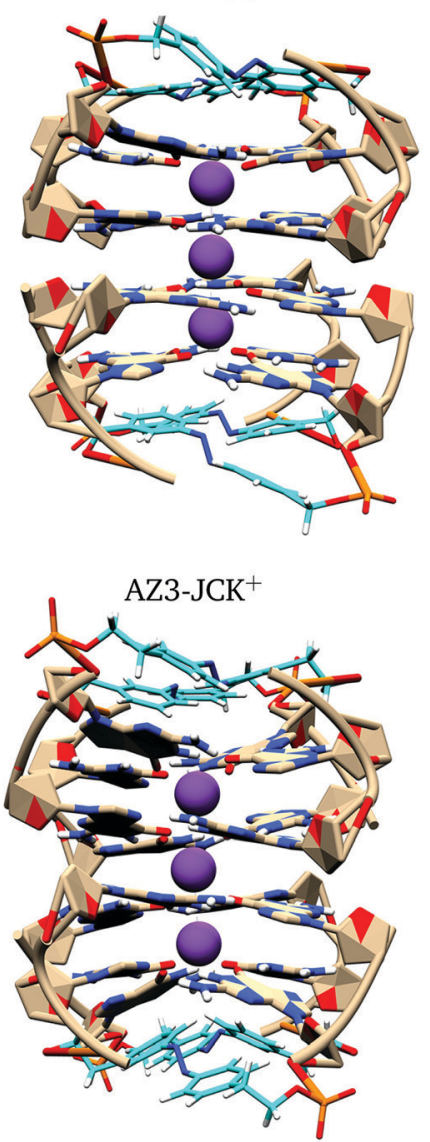

AZ1-JCKCl

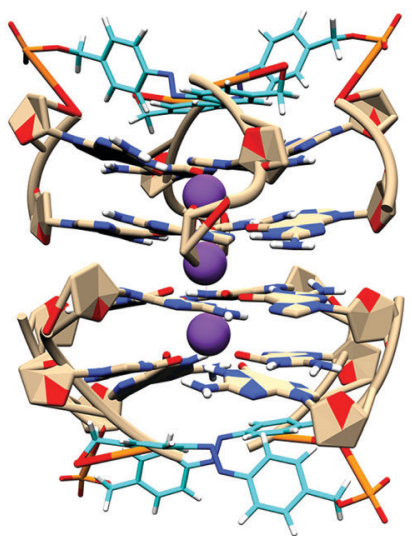

AZ2-JCKCl

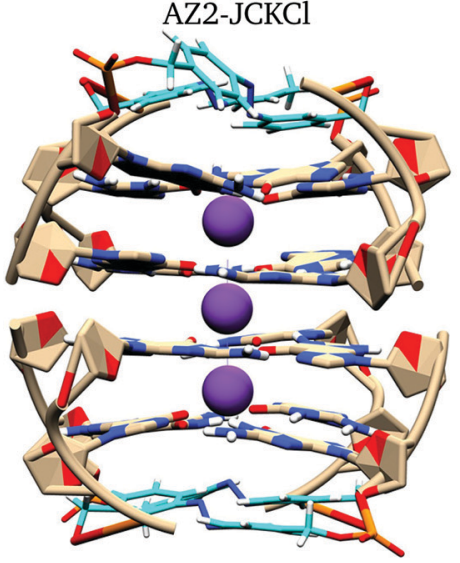

AZ3-JCKCl

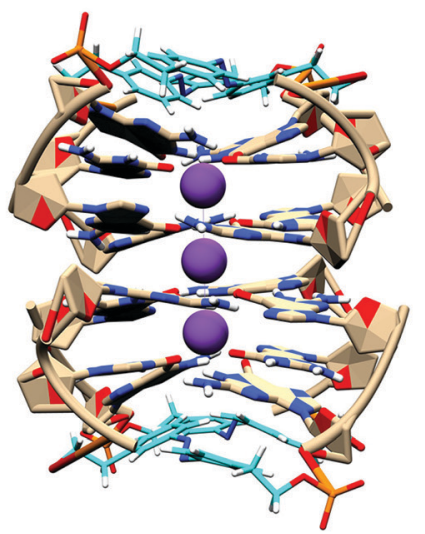

Fig. 3 Representative structures obtained via clustering analysis for (a) AZ1, (b) AZ2 and (c) AZ3 G-quadruplexes using AAK ${ }^{+}, \mathrm{JCK}^{+}$and $\mathrm{JCKCl}$ parameters. The internal ions in the G-quadruplex channel and external ions are represented in purple and green, respectively.

it after around $2 \mathrm{~ns}$, thereby facilitating the expulsion of the $\mathrm{K}^{+}$ from the channel at $34 \mathrm{~ns}$ (see Fig. S4, ESI $\dagger$ ). In contrast to the

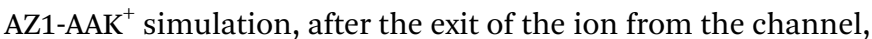
two $\mathrm{K}^{+}$ions from the bulk (shown as green in Fig. 3a) move to align near upper and lower G-quartets until the end of simulation. Interestingly, no ion movement is observed for the AZ1-JCKCl simulation that mimics the experimental conditions (i.e. $100 \mathrm{mM} \mathrm{KCl}$ concentration).
To evaluate the conformational stability of each G-quadruplex using different ion/water parameters, we analyzed the root mean square deviation (RMSD) of these systems along the MD trajectories with respect to the initial structures. In addition, we calculated the RMSDs for the G-quartets and the azobenzene backbone, separately, to understand which part of the G-quadruplex is most affected during the simulations. The RMSD graphs and their average values are presented in Fig. 4 and 
Table 3, respectively. The RMSD graphs for the AZ1 G-quadruplex reveals that despite the fact that the stabilizing ions are very unstable in the AZ1-AAK ${ }^{+}$and $\mathrm{AZ1}_{-}-\mathrm{JCK}^{+}$simulations (see Fig. 3a), the overall system including the G-quartets and azobenzene backbones are relatively stable (Fig. 4a). In contrast, for AZ1JCKCl simulation, an increase in RMSD is observed after around $64 \mathrm{~ns}$ which is mainly attributed to the clockwise rotation of two strands (Fig. 4c). As seen from Table 3, the average values for AZ1JCKCl simulation is $3.80 \AA$ with a standard deviation of 0.83 . As is clear in Fig. 4b, the RMSDs for the azobenzene backbone are notably larger than the those for the G-quartets, which can be attributed to the fact that azobenzene residues that are part of the G-quadruplex backbones are not involved in the stacked G-quartet structures and thus can move freely. Notably, the same behaviour is observed for three independent 200 ns MD runs. Furthermore, the per-atom root mean square fluctuation (RMSF) of the all
Table 3 Average RMSDs $(\AA)$ and their standard deviation values for AZ1, $\mathrm{AZZ}$ and $\mathrm{AZ3} \mathrm{G}$-quadruplexes in $\mathrm{JCKCl}$ simulations

\begin{tabular}{lll}
\hline System & RMSD & Standard deviation \\
\hline AZ1-JCKCl & 3.80 & \pm 0.83 \\
AZ2-JCKCl & 1.94 & \pm 0.48 \\
AZ3-JCKCl & 1.80 & \pm 0.17
\end{tabular}

G-quadruplexes in JCKCl simulations were calculated and plotted in Fig. 5 to understand the structural fluctuation of AZ1, AZ2 and AZ3. According to the RMSF plots, AZ1 fluctuates slightly more than AZ2 and AZ3, showing that AZ1 with high RMSD and RMSF values can adopt two different conformations (see Fig. 5). It is evident that the AZ1 linker, with the para-para substitution pattern, offers a suitable balance between the rigidity and the flexibility of the overall structure that not only allows the (a)

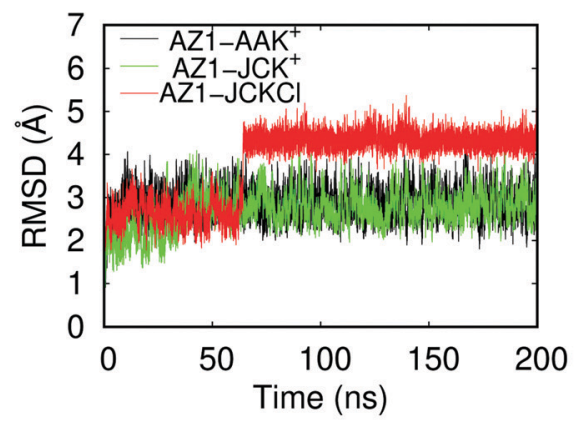

(d)

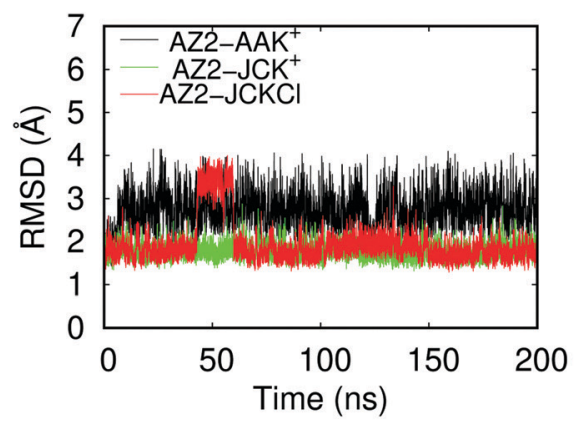

(g)

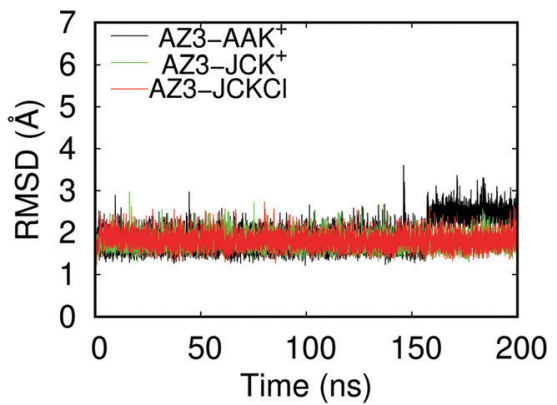

(b)

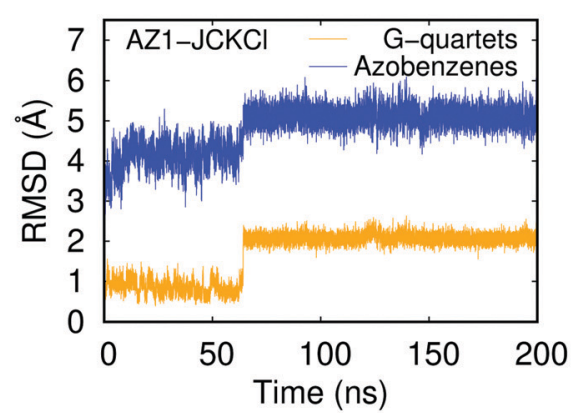

(e)

(h)
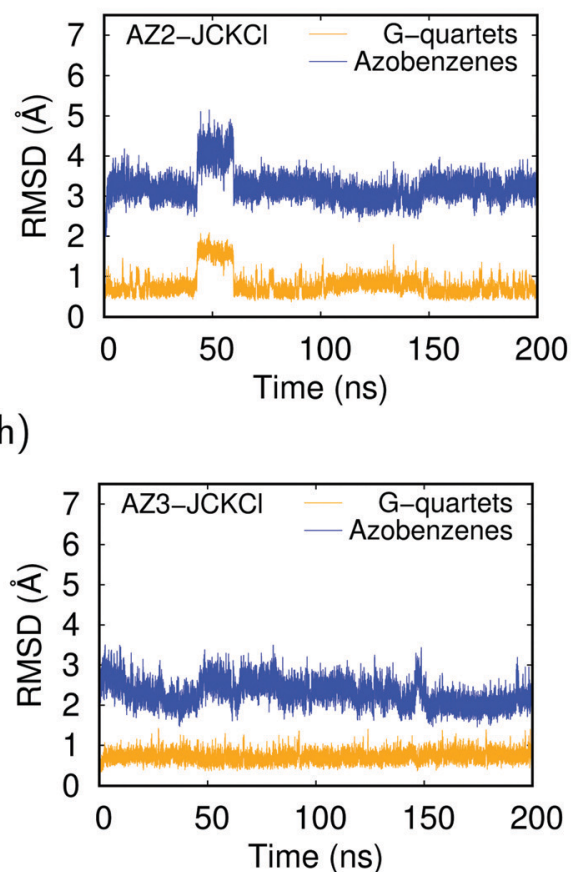

(c) clockwise strand rotation

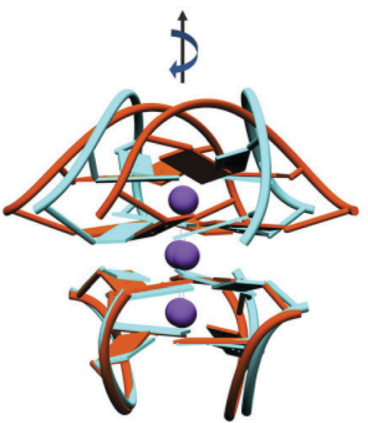

(f)

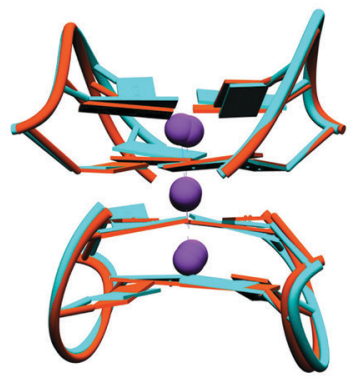

(i)

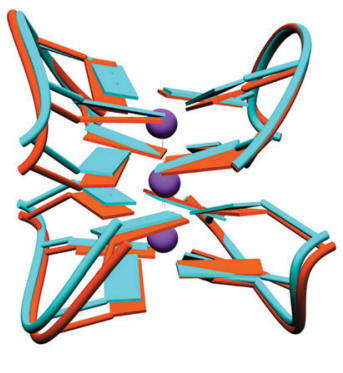

Fig. 4 RMSDs as a function of simulation time. (a, $d$ and g) All atoms of the G-quadruplexes in different simulation groups described in the text. (b, e and h) $\mathrm{G}$-quartets and azobenzenes in $\mathrm{JCKCl}$ simulations. Superimposed structures of $(\mathrm{c})$ the representative structures of the two different ground state populations of the $A Z 1$ and ( $f$ and i) the two random snapshots of $A Z 2$ and $A Z 3$, in JCKCI MD simulations. 


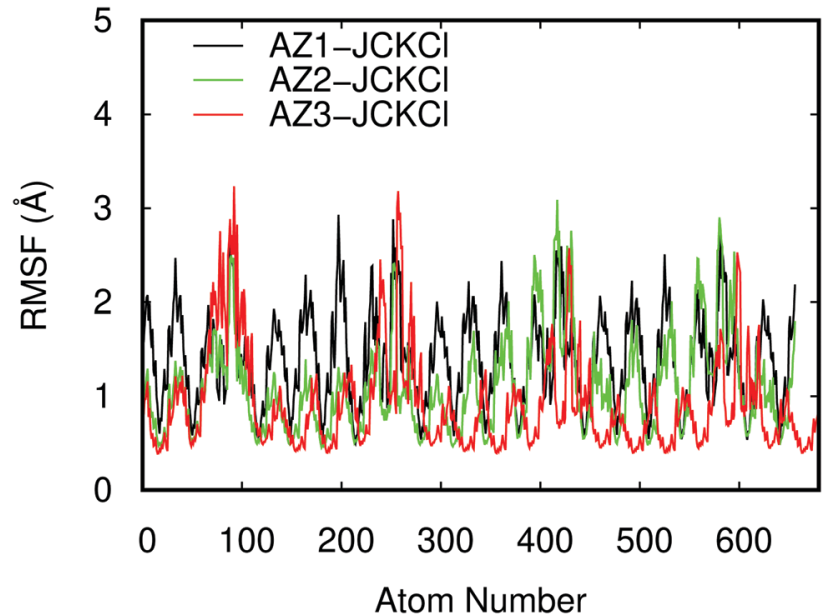

Fig. 5 The per-atom RMSFs of AZ1, AZ2 and AZ3 G-quadruplexes in $\mathrm{JCKCl}$ simulations.

formation of the G-quadruplex but also allows the conformational flexibility of the AZ1 backbone (Fig. 4c).

AZ2 simulations. A similar ion mobility is observed for AZ2$\mathrm{AAK}^{+}$simulation compared to $\mathrm{AZ1}-\mathrm{AAK}^{+}$simulation; however the ion leaves the channel through the bottom G-quartet at around 6 ns of simulation (see Fig. 3b and Fig. S5 for further details, ESI + ). In the $\mathrm{AZ}^{-}-\mathrm{JCK}^{+}$simulation, all three $\mathrm{K}^{+}$ions remain stable within the G-quartets throughout the simulation (Fig. 3b) which is different from AZ1-JCK ${ }^{+}$(Fig. 3a). Similar to the trend observed for AZ1-JCKCl, the ions remain stable during the simulation that mimics the experimental conditions (100 mM KCl concentration). Fig. 4 displays the RMSD plots for the AZ2 G-quadruplex. It is evident that the total RMSD increases from about $2 \AA$ for $\mathrm{AZ}^{-} \mathrm{JCK}^{+}$to about $3 \AA$ for AZ2$\mathrm{AAK}^{+}$which is clearly due to the escape of the ion from the G-quadruplex channel in the AZ2-AAK ${ }^{+}$simulation. The RMSD for the AZ2-JCKCl simulation indicates a jump in the period from 42 to $59 \mathrm{~ns}$ (see Fig. 4e), which is mainly attributed to the deviation of the two strands of the G-quadruplex with respect to the initial reference structure. However, after $60 \mathrm{~ns}$, the RMSD drops and the AZ2 linker and the G-quartets remain stable for the rest of the simulation. Similar to AZ1 simulations, the RMSDs for the azobenzene backbone are notably larger than the those for G-quartets (Fig. 4e). According to the RMSF plot in Fig. 5, AZ2 shows slightly less flexibility compared to the AZ1 in JCKCl simulations.

AZ3 simulations. Under three different simulations, i.e. AZ3$\mathrm{AAK}^{+}, \mathrm{AZ}^{-}-\mathrm{JCK}^{+}$, and $\mathrm{AZ3}-\mathrm{KCl}$ simulations, the $\mathrm{K}^{+}$ions stay stable within G-quartets during the course of the simulations (see Fig. 3c). Similar to AZ2, the RMSD stays stable for AZ3JCKCl simulation that resembles that in the experimental conditions (Fig. 4g). It should be noted that the RMSDs for the azobenzene backbone in the AZ3-JCKCl simulation (Fig. 4h) are smaller that those obtained for AZ1 and AZ2 simulations (Fig. 4b-e), reflecting the smaller variations of the AZ3 backbone compared to AZ1 and AZ2. Furthermore, the RMSF plot in Fig. 5 for AZ3-JCKCl show less flexibility compared to the other two azobenzene derivatives. This means that, despite the fact that the AZ3 backbone possesses a longer side chain (double homologue of the AZ2) and is expected to have more flexibility, the AZ1 and AZ2 backbones, with shorter side chains, undergo larger fluctuations than AZ3, with AZ1 with para-para substitution being the most flexible one.

In sum, it is clear that ion movements and structural stability of the G-quadruplexes are significantly affected by the ion/water parameters. It should be mentioned that using $\mathrm{AAK}^{+}$ion/water parameters shows the escape of ions from the G-quadruplex channel which is an artifact as previously reported for the typical G-quadruplex simulations. ${ }^{88}$ Interestingly, under experimental conditions $(100 \mathrm{mM} \mathrm{KCl}$ concentration) and using the JC parameters, ions remain stable for all three AZ1, AZ2 and AZ3 G-quadruplexes throughout $200 \mathrm{~ns}$ simulations. In addition, the simulations were stable until $500 \mathrm{~ns}$ and we did not observe any changes (see Fig. S7, ESI $\dagger$ ). Our results show that while the AZ2 and AZ3 are structurally stable during the MD runs (see Fig. $4 \mathrm{f}$ and i), for the AZ1 G-quadruplex, we observed two ground state populations that differ by the azobenzene backbone orientations, leading to more conformational changes (see Fig. 4c). Introduction of azobenzene derivatives with the para-para substitution pattern into the G-quadruplex, i.e. AZ1, provides an appropriate balance between the rigidity and the flexibility of the overall structure. The latter can be considered as the main factor favoring photoisomerzation reaction of AZ1 compared to AZ2 and AZ3 between a nonpolymorphic, stacked, tetramolecular G-quadruplex and an unstructured state after trans-cis isomerization occurring in a longer time dynamics, in agreement with experimental findings. ${ }^{23}$ It should be pointed out that the new version of AMBER force field for DNA (parmbsc1) ${ }^{54}$ and ion/ water parameters ${ }^{52,89}$ in future studies might accomplish improved agreement with experiments.

\subsection{QM/MM simulations}

To better understand the effect of the G-quadruplex on the absorption spectra of the azobenzene derivatives, we calculated vertical excitation energies for 90 snapshots, taken from the JCKCl MD simulations, within QM/MM, framework. The average excitation energies of the first two absorption bands $\left(\mathrm{S}_{1}\right.$ and $\left.\mathrm{S}_{2}\right)$ of the azobenzene derivatives in the presence and absence of the point charges of the rest of the DNA are summarized in Table 4 alongside experimental values and the corresponding values for the quantum mechanically optimized isolated azobenzene derivatives. Furthermore, natural transition orbital (NTO) analysis shows that for all the snapshots the $\mathrm{S}_{1}$ is the dark $n \pi^{*}$ state while the $\mathrm{S}_{2}$, with a noticeable oscillator strength, is the bright $\pi \pi^{*}$ (the state-averaged NTO involved in the transitions are shown in Fig. S6, ESI $\dagger$ ). As one can see in Table 4 , the excitation energies of the $S_{1}$ state for the single optimized structures are in perfect agreement with experimental values (difference of about $0.02 \mathrm{eV}$ ) and inclusion of the G-quadruplex environment along with taking the thermal fluctuation into account (average over 90 snapshots) do not significantly affect the excitation energies. The average 
Table 4 Excitation energies calculated for AZ1, AZ2 and AZ3 with and without MM charges using TDDFT( $\omega B$ 97X-D)/cc-pVDZ. The energies are given in eV

\begin{tabular}{llll}
\hline $\mathrm{QM}$ & $\mathrm{MM}$ & $\mathrm{S}_{1}$ & $\mathrm{~S}_{2}$ \\
\hline $\mathrm{AZ1}^{a}$ & - & 2.84 & 4.24 \\
$\mathrm{AZ1}^{b}$ & All & $2.67 \pm 0.23$ & $4.67 \pm 0.38$ \\
$\mathrm{AZ1}^{b}$ & - & $2.65 \pm 0.21$ & $4.77 \pm 0.26$ \\
$\mathrm{AZ2}^{a}$ & - & 2.83 & 4.30 \\
$\mathrm{AZ2}^{b}$ & All & $2.72 \pm 0.19$ & $4.71 \pm 0.40$ \\
$\mathrm{AZ2}^{b}$ & - & $2.72 \pm 0.19$ & $4.70 \pm 0.28$ \\
$\mathrm{AZ3}^{a}$ & - & 2.82 & 4.28 \\
$\mathrm{AZ3}^{b}$ & All & $2.72 \pm 0.19$ & $4.74 \pm 0.29$ \\
$\mathrm{AZ3}^{b}$ & - & $2.72 \pm 0.18$ & $4.70 \pm 0.28$ \\
Experiment $^{c}$ & All & 2.82 & 4.96
\end{tabular}

${ }^{a}$ Using a single gas-phase optimized structure of AZ1, AZ2 and AZ3. ${ }^{b}$ Average energies calculated using QM/MM structures obtained from 90 MD snapshots. ${ }^{c}$ Ref. 23.

excitation energies of the $S_{1}$ state for AZ1 with and without point charges differ by $0.02 \mathrm{eV}$ with a standard deviation about $0.2 \mathrm{eV}$. In the case of AZ2 and AZ3, the inclusion of the point charge environment does not change the average excitation energies of $\mathrm{S}_{1}$ state $(2.72 \mathrm{eV}$ with a standard deviation about $0.2 \mathrm{eV})$. However, the excitation energies of the $S_{2}$ state $\left(\pi \pi^{*}\right)$ for the single optimized structures are smaller than the corresponding experimental values (4.24, 4.20 and $4.28 \mathrm{eV}$ for AZ1, $\mathrm{AZ2}$ and $\mathrm{AZ3}$, respectively, vs. $4.96 \mathrm{eV}$ ). Interestingly, taking only the thermal fluctuation into account (QM/MM average energies using 90 snapshots), without the inclusion of the G-quadruplex environment, blue-shifts the $\mathrm{S}_{2}$ excitation energies relative to the gas-phase value $(\approx 0.35 \mathrm{eV})$, thus getting closer to the experimental value. Furthermore, the inclusion of the G-quadruplex environment, does not have a significant effect on the $\mathrm{S}_{2}$ excitation energies relative to the average energies calculated using structures obtained from $90 \mathrm{MD}$ snapshots (e.g. 4.71 vs. 4.70 for AZ2), implying that the influence of the environment, presented by fixed point charges, in the $S_{2}$ excitation energies is minimal. We propose that the latter can be attributed to the position of the azobenzene linkers in the G-quadruplexes (see Fig. 1), i.e. the edgewise loops containing the azobenzene moieties that are located above the G-quartets, not being fully embedded inside or being involved in the stacked structure. In sum, the average excitation energies of the $\mathrm{S}_{2}$ state for AZ1, AZ2 and AZ3 is about $4.7 \mathrm{eV}$ with standard deviation $0.3-0.4$ confirming the broad distribution of the peak maxima in the absorption spectra.

The experimental findings ${ }^{23}$ show that AZ1, AZ2 and AZ3 G-quadruplexes have a similar absorption spectrum (red in Fig. 6), that exhibits two bands, the less intense band $\left(S_{1}\right)$ corresponding to $2.82 \mathrm{eV}(440 \mathrm{~nm})$ and the most intense band $\left(\mathrm{S}_{2}\right)$ at $4.96 \mathrm{eV}(250 \mathrm{~nm})$. Here, the resulting excitation energies were convoluted with Gaussian of suitable full width at half maximum (FWHM) of the corresponding experimental spectrum, to account for instrumental resolution and other broadening effects that are not accounted in our MD snapshots. The calculated spectra are plotted in Fig. 6. The comparison between theory and experiment shows a very satisfactory

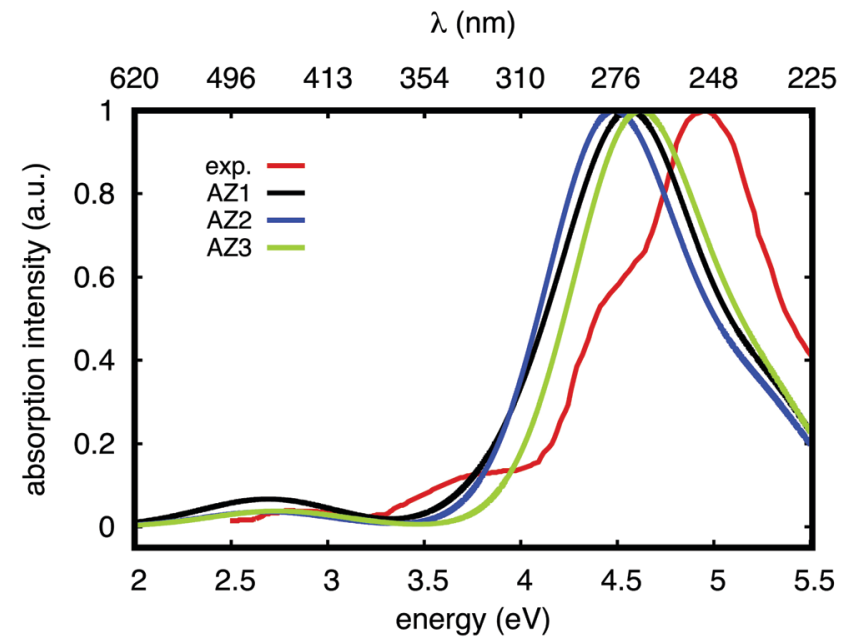

Fig. 6 Absorption spectra of AZ1, AZ2 and AZ3 obtained by a Gaussian convolution of the excitation energies of 90 MD simulation snapshots. The experimental spectrum is shown in red which is taken from ref. 23.

qualitative, partly quantitative, agreement. In the computed spectra for AZ1, AZ2 and AZ3, two peaks are present, but they are red-shifted by about $0.1 \mathrm{eV}$ and $0.5 \mathrm{eV}$ for $\mathrm{S}_{1}$ and $\mathrm{S}_{2}$, respectively compared to the corresponding experimental peaks.

\section{Conclusions}

In summary, we investigated the effect of the size and substitution pattern of the azobenzene derivatives on their spectroscopic properties within the smallest G-quadruplex structure using hybrid quantum classical simulations.

We applied the SF-TDDFT method to explore the photoisomerization mechanism of the azobenzene derivatives in the gas phase. The calculations reveal that all three derivatives have similar photoisomerization reactions which occur via three consecutive steps; (i) $\mathrm{S}_{0} \rightarrow \mathrm{S}_{2}$ excitation, (ii) rapid decay from $\mathrm{S}_{2}$ to $\mathrm{S}_{1}$ passing the $\mathrm{CI}_{\mathrm{S}_{2} / \mathrm{S}_{1}}$, (iii) decay to the ground state of the trans or cis isomer via $\mathrm{CI}_{\mathrm{S}_{1} / \mathrm{s}_{0}}$. The similar photodynamics observed for the AZ1, AZ2, and AZ3 derivatives indicates that the size and the substitution pattern do not affect significantly the ultra-fast cis-trans photoiomerization mechanism of the azobenzene unit, in line with the experimental observation.

The MD simulations performed under different ion/water parameters and concentrations revealed that the structural stability of the G-quadruplex and the ion mobility in the channel are very sensitive to the these parameters. Using the combination of Åqvist parameters for the $\mathrm{K}^{+}$and TIP3P water models $\left(\mathrm{AAK}^{+}\right)$, we observed the escape of ions from the G-quadruplex channel which is not in the agreement with the reported ion residence lifetime. ${ }^{90,91}$ With the same water model, using the JC ion parameter under $100 \mathrm{mM} \mathrm{KCl}$ concentration (JCKCl), i.e. experimental conditions, the $\mathrm{K}^{+}$ions remain tightly bound in the G-quadruplex channel during the simulations. Moreover, under JCKCl conditions, AZ2 and AZ3 G-quadruplex are structurally stable during the simulations, while AZ1 shows two ground state populations that differ by the 
azobenzene backbone adopting two different conformations, leading to more conformational variation. In fact, introducing the azobenzene derivative with para-para substitution pattern into the G-quadruplex (i.e. AZ1) provides more flexibility to the structure compared to AZ2 and AZ3 and can thus facilitate the photoisomerization reaction between a nonpolymorphic, stacked, tetramolecular G-quadruplex and an unstructured state after trans-cis isomerization occurring in a longer time dynamics, in agreement with experimental findings. ${ }^{23}$

The simulation of the absorption spectra of the azobenzene derivatives within the $\mathrm{QM} / \mathrm{MM}$ framework showed that the thermal fluctuation plays a more significant role in the excitation energy of the $S_{2}$ than the inclusion of the G-quadruplex, implying that the influence of the environment, presented by fixed point charges, is minimal. We suggest that the latter can be attributed to the position of the azobenzene linkers in the G-quadruplexes, i.e. the edgewise loops containing the azobenzene moieties that are located above the G-quartets, not being involved in the stacked structure. Our theoretical findings provide atomistic insights into the recent experimental study of the photoresponsive formation of photoswitchable G-quadruplex motifs at the atomic level, thus providing design principles for developing azobenzene-based photocontrollable DNA G-quarduplexes relevant for novel nanodevices with medical and nanotechnology applications. It would be very important to study the photoisomerization reactions of azobenzene derivatives within G-quadruplex and work in this direction is in progress in our group.

\section{Conflicts of interest}

There are no conflicts to declare.

\section{Acknowledgements}

This work is part of Innovational Research Incentives Scheme Vidi 2017 with project number 016.Vidi.189.044, which is (partly) financed by the Dutch Research Council (NWO).

\section{Notes and references}

1 S. Burge, G. N. Parkinson, P. Hazel, A. K. Todd and S. Neidle, Nucleic Acids Res., 2006, 34, 5402-5415.

2 S. Neidle, Curr. Opin. Struct. Biol., 2009, 19, 239-250.

3 S. Neidle and S. Balasubramanian, Quadruplex nucleic acids, Royal Society of Chemistry, 2006, vol. 7.

4 R. Hänsel-Hertsch, M. Di Antonio and S. Balasubramanian, Nat. Rev. Mol. Cell Biol., 2017, 18, 279-284.

5 S. Neidle, FEBS J., 2010, 277, 1118-1125.

6 M. L. Bochman, K. Paeschke and V. A. Zakian, Nat. Rev. Genet., 2012, 13, 770.

7 H. J. Lipps and D. Rhodes, Trends Cell Biol., 2009, 19, 414-422.

8 A. Ou, J. W. Schmidberger, K. A. Wilson, C. W. Evans, J. A. Hargreaves, M. Grigg, M. L. OMara, K. S. Iyer, C. S. Bond and N. M. Smith, Nucleic Acids Res., 2020, 48, 5766-5776.
9 K. G. Moghaddam, A. H. de Vries, S. J. Marrink and S. Faraji, Biophys. Chem., 2019, 253, 106220.

10 F. Wang, X. Liu and I. Willner, Angew. Chem., Int. Ed., 2015, 54, 1098-1129.

11 M. McCullagh, I. Franco, M. A. Ratner and G. C. Schatz, J. Am. Chem. Soc., 2011, 133, 3452-3459.

12 X. Wang, J. Huang, Y. Zhou, S. Yan, X. Weng, X. Wu, M. Deng and X. Zhou, Angew. Chem., Int. Ed., 2010, 49, 5305-5309.

13 D. Miyoshi, S. Matsumura, W. Li and N. Sugimoto, Nucleosides, Nucleotides Nucleic Acids, 2003, 22, 203-221.

14 T. Li, S. Dong and E. Wang, J. Am. Chem. Soc., 2010, 132, 13156-13157.

15 D. M. Engelhard, J. Nowack and G. H. Clever, Angew. Chem., Int. Ed., 2017, 56, 11640-11644.

16 D. P. Gonçalves, R. Rodriguez, S. Balasubramanian and J. K. Sanders, Chem. Commun., 2006, 4685-4687.

17 D. P. Gonçalves, S. Ladame, S. Balasubramanian and J. K. Sanders, Org. Biomol. Chem., 2006, 4, 3337-3342.

18 R. Rodriguez, G. D. Pantos, D. P. Gonçalves, J. K. Sanders and S. Balasubramanian, Angew. Chem., Int. Ed., 2007, 46, 5405-5407.

19 A. S. Lubbe, W. Szymanski and B. L. Feringa, Chem. Soc. Rev., 2017, 46, 1052-1079.

20 W. Szymanski, J. M. Beierle, H. A. Kistemaker, W. A. Velema and B. L. Feringa, Chem. Rev., 2013, 113, 6114-6178.

21 S. Lena, P. Neviani, S. Masiero, S. Pieraccini and G. P. Spada, Angew. Chem., Int. Ed., 2010, 49, 3657-3660.

22 S. Ogasawara and M. Maeda, Angew. Chem., Int. Ed., 2009, 48, 6671-6674.

23 J. Thevarpadam, I. Bessi, O. Binas, D. P. Gonçalves, C. Slavov, H. R. Jonker, C. Richter, J. Wachtveitl, H. Schwalbe and A. Heckel, Angew. Chem., Int. Ed., 2016, 55, 2738-2742.

24 H. D. Bandara and S. C. Burdette, Chem. Soc. Rev., 2012, 41, 1809-1825.

25 T. Schultz, J. Quenneville, B. Levine, A. Toniolo, T. J. Martnez, S. Lochbrunner, M. Schmitt, J. P. Shaffer, M. Z. Zgierski and A. Stolow, J. Am. Chem. Soc., 2003, 125, 8098-8099.

26 M. Böckmann, N. L. Doltsinis and D. Marx, J. Phys. Chem. A, 2010, 114, 745-754.

27 C. Ciminelli, G. Granucci and M. Persico, Chem. - Eur. J., 2004, 10, 2327-2341.

28 A. Toniolo, C. Ciminelli, M. Persico and T. Martnez, J. Chem. Phys., 2005, 123, 234308.

29 M. Böckmann, C. Peter, L. D. Site, N. L. Doltsinis, K. Kremer and D. Marx, J. Chem. Theory Comput., 2007, 3, 1789-1802.

30 I. Conti, M. Garavelli and G. Orlandi, J. Am. Chem. Soc., 2008, 130, 5216-5230.

31 T. Cusati, G. Granucci and M. Persico, J. Am. Chem. Soc., 2011, 133, 5109-5123.

32 O. Weingart, Z. Lan, A. Koslowski and W. Thiel, J. Phys. Chem. Lett., 2011, 2, 1506-1509.

33 J. A. Gamez, O. Weingart, A. Koslowski and W. Thiel, J. Chem. Theory Comput., 2012, 8, 2352-2358.

34 Y. Harabuchi, M. Ishii, A. Nakayama, T. Noro and T. Taketsugu, J. Chem. Phys., 2013, 138, 064305.

35 M. Pederzoli, J. Pittner, M. Barbatti and H. Lischka, J. Phys. Chem. A, 2011, 115, 11136-11143. 
36 T. Pancur, F. Renth, F. Temps, B. Harbaum, A. Krüger, R. Herges and C. Näther, Phys. Chem. Chem. Phys., 2005, 7, 1985-1989.

37 A. Cembran, F. Bernardi, M. Garavelli, L. Gagliardi and G. Orlandi, J. Am. Chem. Soc., 2004, 126, 3234-3243.

38 P. Tavadze, G. Avendanìfo Franco, P. Ren, X. Wen, Y. Li and J. P. Lewis, J. Am. Chem. Soc., 2018, 140, 285-290.

39 D. Rastädter, M. Biswas and I. Burghardt, J. Phys. Chem. B, 2014, 118, 8478-8488.

40 M. Biswas and I. Burghardt, Biophys. J., 2014, 107, 932-940.

41 D. Wu, Y.-T. Wang, W.-H. Fang, G. Cui and W. Thiel, Chem. - Asian J., 2018, 13, 780-784.

42 S.-H. Xia, G. Cui, W.-H. Fang and W. Thiel, Angew. Chem., 2016, 128, 2107-2112.

43 P. Mondal, G. Granucci, D. Rastädter, M. Persico and I. Burghardt, Chem. Sci., 2018, 9, 4671-4681.

44 A. Pérez, I. Marchán, D. Svozil, J. Sponer, T. E. Cheatham, C. A. Laughton and M. Orozco, Biophys. J., 2007, 92, 3817-3829.

45 M. P. Long, S. Alland, M. E. Martin and C. M. Isborn, Phys. Chem. Chem. Phys., 2020, 22, 5584-5596.

46 H. W. Kim, Y. M. Rhee and S. K. Shin, Phys. Chem. Chem. Phys., 2018, 20, 21068-21074.

47 A. K. Sahoo, B. Bagchi and P. K. Maiti, J. Chem. Phys., 2019, 151, 164902.

48 J. Wang, R. M. Wolf, J. W. Caldwell, P. A. Kollman and D. A. Case, J. Comput. Chem., 2004, 25, 1157-1174.

49 T. Fox and P. A. Kollman, J. Phys. Chem. B, 1998, 102, 8070-8079.

50 J. Aqvist, J. Phys. Chem., 1990, 94, 8021-8024.

51 W. L. Jorgensen, J. Chandrasekhar, J. D. Madura, R. W. Impey and M. L. Klein, J. Chem. Phys., 1983, 79, 926-935.

52 I. S. Joung and T. E. Cheatham III, J. Phys. Chem. B, 2008, 112, 9020-9041.

53 H. Berendsen, J. Grigera and T. Straatsma, J. Phys. Chem., 1987, 91, 6269-6271.

54 M. Havrila, P. Stadlbauer, B. Islam, M. Otyepka and J. SìOEponer, J. Chem. Theory Comput., 2017, 13, 3911-3926.

55 G. Bussi, D. Donadio and M. Parrinello, J. Chem. Phys., 2007, 126, 014101.

56 H. J. Berendsen, J. v. Postma, W. F. van Gunsteren, A. DiNola and J. R. Haak, J. Chem. Phys., 1984, 81, 3684-3690.

57 M. Parrinello and A. Rahman, J. Appl. Phys., 1981, 52, 7182-7190.

58 S. Nosé and M. Klein, Mol. Phys., 1983, 50, 1055-1076.

59 U. Essmann, L. Perera, M. L. Berkowitz, T. Darden, H. Lee and L. G. Pedersen, J. Chem. Phys., 1995, 103, 8577-8593.

60 B. Hess, H. Bekker, H. J. Berendsen and J. G. Fraaije, J. Comput. Chem., 1997, 18, 1463-1472.

61 B. Hess, C. Kutzner, D. Van Der Spoel and E. Lindahl, J. Chem. Theory Comput., 2008, 4, 435-447.

62 X. Daura, K. Gademann, B. Jaun, D. Seebach, W. F. Van Gunsteren and A. E. Mark, Angew. Chem., Int. Ed., 1999, 38, 236-240.
63 Y. Shao, Z. Gan, E. Epifanovsky, A. T. Gilbert, M. Wormit, J. Kussmann, A. W. Lange, A. Behn, J. Deng and X. Feng, et al., Mol. Phys., 2015, 113, 184-215.

64 A. I. Krylov and P. M. Gill, Wiley Interdiscip. Rev.: Comput. Mol. Sci., 2013, 3, 317-326.

65 H. M. Senn and W. Thiel, Angew. Chem., Int. Ed., 2009, 48, 1198-1229.

66 Y. Shao, M. Head-Gordon and A. I. Krylov, J. Chem. Phys., 2003, 118, 4807-4818.

67 T. H. Dunning Jr, J. Chem. Phys., 1989, 90, 1007-1023.

68 P. Hohenberg and W. Kohn, Phys. Rev., 1964, 136, B864.

69 W. Kohn and L. J. Sham, Phys. Rev., 1965, 140, A1133.

70 J.-D. Chai and M. Head-Gordon, Phys. Chem. Chem. Phys., 2008, 10, 6615-6620.

71 S. Grimme, J. Comput. Chem., 2006, 27, 1787-1799.

72 S. Maeda, K. Ohno and K. Morokuma, J. Chem. Theory Comput., 2010, 6, 1538-1545.

73 K. D. Closser, O. Gessner and M. Head-Gordon, J. Chem. Phys., 2014, 140, 134306.

74 D. L. Beveridge and H. Jaffe, J. Am. Chem. Soc., 1966, 88, 1948-1953.

75 J. Bouwstra, A. Schouten and J. Kroon, Acta Crystallogr., Sect. C: Cryst. Struct. Commun., 1983, 39, 1121-1123.

76 T. Tsuji, H. Takashima, H. Takeuchi, T. Egawa and S. Konaka, J. Phys. Chem. A, 2001, 105, 9347-9353.

77 E. V. Brown and G. R. Granneman, J. Am. Chem. Soc., 1975, 97, 621-627.

78 L. Yue, Y. Liu and C. Zhu, Phys. Chem. Chem. Phys., 2018, 20, 24123-24139.

79 L. Yu, C. Xu and C. Zhu, Phys. Chem. Chem. Phys., 2015, 17, 17646-17660.

80 L. Yu, C. Xu, Y. Lei, C. Zhu and Z. Wen, Phys. Chem. Chem. Phys., 2014, 16, 25883-25895.

81 T. Ishikawa, T. Noro and T. Shoda, J. Chem. Phys., 2001, 115, 7503-7512.

82 P. Cattaneo and M. Persico, Phys. Chem. Chem. Phys., 1999, 1, 4739-4743.

83 J. Casellas, M. J. Bearpark and M. Reguero, ChemPhysChem, 2016, 17, 3068-3079.

84 H. Fliegl, A. Köhn, C. Hättig and R. Ahlrichs, J. Am. Chem. Soc., 2003, 125, 9821-9827.

85 J.-Å. Andersson, R. Petterson and L. Tegnér, J. Photochem., 1982, 20, 17-32.

86 C. Xu, L. Yu, F. L. Gu and C. Zhu, Phys. Chem. Chem. Phys., 2018, 20, 23885-23897.

87 M. Rebič, A. Laaksonen, J. Sponer, J. Uličný and F. Mocci, J. Phys. Chem. B, 2016, 120, 7380-7391.

88 M. RebicìOE, A. Laaksonen, J. Sponer, J. Uličný and F. Mocci, J. Phys. Chem. B, 2016, 120, 7380-7391.

89 S. Mamatkulov and N. Schwierz, J. Chem. Phys., 2018, 148, 074504.

90 D. Bhattacharyya, G. Mirihana Arachchilage and S. Basu, Front. Chem., 2016, 4, 38.

91 E. Largy, J.-L. Mergny and V. Gabelica, The Alkali Metal Ions: Their Role for Life, Springer, 2016, pp. 203-258. 